\title{
El papel de la orientación al aprendizaje en la innovación y el desempeño: Un estudio en micro, pequeñas y medianas empresas en San Luis Potosí (México)
}

\author{
The role of learning orientation in innovation and business performance: \\ a case study in micro, small and medium firms in San Luis Potosi (Mexico)
}

\author{
María del Pilar Pastor Pérez ${ }^{1 *}$, Paola Isabel Rodríguez Gutiérrez ${ }^{1}$, \\ Jesús Collado Agudob ${ }^{2}$ \\ ${ }^{I}$ Universidad Autónoma de San Luis Potosí, México \\ ${ }^{2}$ Universidad de Cantabria, España
}

Recibido el 14 de septiembre de 2017; aceptado el 17 de octubre de 2018

Disponible en Internet el: 9 de noviembre de 2018

\section{Resumen}

Este trabajo presenta los resultados de un estudio empírico en el que se evalúa el papel de la orientación al aprendizaje en el enfoque a la innovación y el desempeño de micro, pequeñas y medianas empresas. Además, se analiza si la edad y el tamaño de la organización influyen en la relación entre aprendizaje e innovación. El método utilizado para la recogida de datos fue el de encuesta a una muestra de 253 empresas de los sectores industria, comercio y servicios en México, en la ciudad de San Luis Potosí. Se estimó un modelo de ecuaciones estructurales y los hallazgos indican que la orientación al aprendizaje influye de manera positiva sobre el desempeño empresarial y el enfoque a la innovación de las empresas de la muestra. Se observa también que el tamaño de la empresa tiene un efecto positivo sobre el enfoque a la innovación; sin embargo, no se encuentra efecto significativo de la edad de la organización en la innovación. Estas conclusiones contribuyen a incrementar la evidencia sobre capacidades de las empresas de menor tamaño.

Códigos JEL: M10, M 20, O30

Palabras clave: Orientación al aprendizaje; Enfoque a la innovación; Desempeño empresarial.

*Autor para correspondencia.

Correo electrónico: pilar.pastor@uaslp.mx (M. P. Pastor Pérez)

La revisión por pares es responsabilidad de la Universidad Nacional Autónoma de México. 


\begin{abstract}
This paper presents the results of an empirical study that evaluates the role of learning orientation in business performance and innovation of micro, small and medium enterprises. In addition, it is analyzed if the age and size of the organization influence the relationship between learning and innovation. The empirical data were drawn from a sample of 253 companies in the industry, commerce and services sectors in Mexico, in the city of San Luis Potosí. A model of structural equations was estimated and the findings indicate that the learning orientation has a positive influence on performance and innovation orientation of the companies. It is also observed that the size of the company has a positive effect on innovation; however, no significant effect of the age of the organization on innovation is found. These conclusions contribute to increasing the evidence on the capabilities of smaller companies.
\end{abstract}

JEL Codes: M10, M 20, O30

Keywords: Learning orientation; Innovation; Firm performance.

\title{
Introducción
}

Las competencias y capacidades distintivas de las organizaciones dependen de diversos factores que según Teece, Pisano y Shuen (1997) se agrupan en tres categorías: recursos o activos particulares, trayectoria de la organización, y procesos organizacionales y de gestión, entre los que destaca el aprendizaje. Además, en entornos cada vez más cambiantes, la teoría de capacidades dinámicas reconoce que las empresas tienen que ir renovando sus capacidades y para ello la adaptación, absorción e innovación son factores esenciales (Wang y Ahmed, 2007). A la vez, ser capaz de aprender es clave, en especial, para la innovación que tiene una gran exigencia en términos de generación y aplicación de conocimiento (Senge, Roberts, Ross, Smith y Kleiner, 1994). En esta lógica, cabe esperar que las organizaciones que gestionan adecuadamente el proceso de aprendizaje, incluyendo la adquisición de conocimiento, la distribución e interpretación de información, y la memoria organizacional, son capaces de desarrollar ventajas competitivas (Huber, 1991) que repercuten en sus indicadores de desempeño.

La evidencia empírica sobre la relación entre las capacidades dinámicas y el rendimiento depende del tipo de capacidades observadas — genéricas o específicas—, de la inclusión en los modelos de variables procedentes del entorno, y de los métodos de medición o análisis utilizados (Pezeshkan, Fainshmidt, Nairm Frazier y Markowski, 2016). Sin embargo, en las empresas más orientadas al aprendizaje se suelen observar relaciones positivas entre el aprendizaje, los resultados y supervivencia organizacionales y también con la innovación (Keskin, 2006; Alegre y Chiva, 2008, 2013; Torres y Jasso, 2009; Rhee, Park y Lee, 2010; Wang y Ellinger, 2011; Sok, O’Cass y Sok, 2013; Gomes y Wojahn, 2017; Liu, 2017; Tajeddini, Altinay y Ratten, 2017).

En México, como en otras economías emergentes, los resultados promedio de innovación y desempeño empresarial no son alentadores ${ }^{1}$ y esta situación es más crítica entre las empresas

\footnotetext{
${ }^{1}$ Según OECD (2017, 43), la productividad laboral de México en 2015 fue la segunda menor, de entre 39 países comparados, solo mayor que la de Costa Rica. Además, su crecimiento entre 1995-2015 es el tercero más bajo, después de Italia y España. Aproximadamente, en el $95 \%$ de las empresas trabajan menos de cinco empleados, mientras que en Estados Unidos este indicador está en torno al 40\%. Dichas empresas emplean hasta el $40 \%$ de la fuerza laboral y poseen el $15 \%$ del capital de la economía, pero generan poco valor agregado, por lo que tienen una productividad muy baja (Cavallo
} 
de menor tamaño. Las causas de dicha realidad son multifacéticas, pero detonar procesos de aprendizaje en las organizaciones debería tener impactos positivos a nivel micro y macroeconómico.

En este sentido, las investigaciones sobre las consecuencias del aprendizaje en la innovación de las empresas mexicanas de tamaño micro, pequeño y mediano son pocas, y algunas de ellas se centran en sectores económicos de características muy particulares, como podemos observar a continuación.

Mendoza y Valenzuela (2014) analizan el aprendizaje, la innovación y la gestión tecnológica en micro, pequeñas y medianas empresas de la industria metalmecánica y de tecnologías de la información. Los autores concluyen que existe una relación intrínseca entre el conocimiento técnico y su gestión; y afirman que, en un marco de aprendizaje, esta relación genera innovación tecnológica. Estrada y Dutrénit (2007) estudian la gestión del conocimiento en micro y pequeñas empresas de maquinados de dos ciudades de México. Encontraron que el capital humano, expresado a través de variables de aprendizaje tácito e individual, ejerce una influencia positiva sobre casi todos los indicadores de innovación; por lo que sugieren que no hay proceso de cambio o mejora sin la intervención de la habilidad, la experiencia y el conocimiento de los recursos humanos; de manera general, sus resultados reafirman la influencia del capital intelectual en el desempeño.

Los trabajos de Martínez Serna, Vega Martínez y Vega Martínez (2016) y Martínez Serna, Vega Martínez y Eternod Domenech (2018) en micro, pequeñas y medianas empresas manufactureras, comerciales y de servicios de la ciudad de Aguascalientes, exploran las relaciones entre la orientación al aprendizaje, el compromiso organizacional, la innovación y el desempeño concluyendo que la orientación al aprendizaje es un buen predictor para mejorar innovación y desempeño empresarial. Sin embargo, no encuentran una relación directa entre la orientación al aprendizaje y el desempeño, sino de manera indirecta a través de la innovación.

San Luis Potosí es uno de los estados mexicanos cuyo crecimiento en los últimos años está por encima del promedio nacional y su tasa de desempleo es menor; sin embargo, no existen trabajos que analicen la capacidad de innovación de la planta productiva centrando la atención en el aprendizaje y la orientación a la innovación de las empresas del estado. ${ }^{2}$

Hablando en particular del desempeño de las micro, pequeñas y medianas empresas, Ndiaye, Razak, Nagayev y Ng (2018) señalan que a pesar de que se ha acrecentado la investigación sobre el desempeño de estas empresas, no hay consenso sobre los factores que lo impulsan y hacen énfasis en que la literatura académica se enfoca desproporcionadamente en economías desarrolladas.

Derivado de lo anterior, en el marco de la teoría de capacidades dinámicas, la pregunta de investigación que subyace en este trabajo es ¿cuál es el papel de la orientación al aprendizaje en la innovación y el desempeño de micro, pequeñas y medianas empresas de la ciudad de San

y Powell, 2018, 34). En cuanto a innovación, la inversión en investigación y desarrollo sigue siendo de las más bajas y en 2016 apenas superó el 0.5\% del PIB, mientras que el promedio de la OCDE fue 2.35\% (OECD, 2018); el número de patentes por millón de habitantes es inferior al que, en promedio, se observa en países de la misma gama de PIB per cápita (Cavallo y Powell, 2018, 36).

${ }^{2}$ Algunos de los trabajos sobre las empresas del estado de San Luis Potosí son los siguientes: Pastor (2012) describe el sistema de innovación del estado; Rodríguez, Fuentes y Rodríguez (2013) estudian las capacidades estratégicas y el desempeño de empresas propiedad de mujeres; Segura, Borjas y Sifuentes (2014) evalúan el ambiente innovador; Rodríguez, Ramos y Pastor (2015) analizan la orientación al mercado en las MIPYMES; Pastor, Rodríguez y Ramos (2017) exploran la adicionalidad generada en micro y pequeñas empresas beneficiarias de financiamiento público para la innovación. 
Luis Potosí? La motivación de la misma es incrementar la evidencia empírica sobre el tema, desde el contexto poco explorado de una economía emergente como México, y las empresas de menor tamaño de los sectores comercio, industria y servicios.

A continuación, a partir de la revisión de la literatura, se justifican las hipótesis y el modelo teórico propuesto. Posteriormente, se explica el método utilizado para dar paso a los resultados. El último epígrafe expone las conclusiones y limitaciones.

\section{Revisión de la literatura y desarrollo de hipótesis}

\section{Orientación al aprendizaje y sus dimensiones}

Esta investigación parte del aprendizaje como un proceso fundamental para la creación de conocimiento que se convierte en un activo fundamental para que las empresas puedan crear valor superior, complejo de desarrollar, y difícil de imitar en mercados dinámicos (Slater y Narver, 1995). Aunque DiBella, Nevis y Gould (1996) afirman que las organizaciones son sistemas sociales en los que, por su propia naturaleza, se aprende, exista o no una estrategia para ello, el aprendizaje relacionado con el nuevo conocimiento especializado es un proceso complejo en el que los miembros de la organización deben buscar posibles errores en la compañía, identificar soluciones y poner en marcha acciones correctivas, compartiendo el conocimiento de manera que pueda ser utilizado por personas diferentes a aquellas que lo desarrollaron (Sinkula, Baker y Noordewier, 1997).

Las organizaciones orientadas al aprendizaje tienen la necesidad y curiosidad de adquirir y comprender nuevas ideas (Hurley y Hult, 1998), deben decidir qué información hay que recopilar, cómo se evaluará e interpretará, cómo se pondrá a disposición de los miembros de la empresa y quién tendrá acceso a ella. Es decir, la orientación al aprendizaje de una compañía consiste en la habilidad de crear conocimiento, diseminarlo y utilizarlo (Liu, Luo y Shi, 2002). Todo esto implica que el aprendizaje relevante no se genera si no existe un correcto sistema organizacional de información compartida, que fluya correctamente por todos los niveles y que permita la revisión de acciones pasadas (Calantone, Cavusgil y Zhao, 2002). En este mismo sentido, Dutrénit (2000) menciona que la administración activa del aprendizaje dinámico incluye mecanismos específicos para gestionar conocimiento tácito y codificado y convertir conocimiento individual en organizacional.

Calantone et al. (2002) afirman que no existe consenso sobre cómo definir y operacionalizar el constructo orientación al aprendizaje; ellos lo definen como la actividad de la organización relativa a la creación y uso de conocimiento para potenciar la ventaja competitiva, y lo conciben compuesto por cuatro dimensiones: compromiso con el aprendizaje, visión compartida, mentalidad abierta, y conocimiento intra-organizacional compartido. A continuación, se explica cada uno de estos componentes, los cuales reflejan los valores necesarios para que la organización desarrolle la capacidad de aprendizaje (Fraj, Matute y Melero, 2013).

\section{Compromiso con el aprendizaje}

La existencia del compromiso al aprendizaje en la compañía depende de qué tan importante sea el aprendizaje para la misma; si no es valorado, seguramente no se promoverá y será escaso (Sinkula et al., 1997). En la situación contraria, el aprendizaje será para la empresa una inversión indispensable para su sobrevivencia (Calantone et al., 2002). Según Wang y Wei (2005), el compromiso al aprendizaje "hace que una empresa esté dispuesta a aprender, y 
aprender aumenta sus habilidades de explorar lo desconocido e identificar nuevas soluciones en contextos inciertos, lo que es esencial para utilizar la inteligencia de mercado a fin de ganar una ventaja competitiva en ambientes de negocios turbulentos." (p. 1164).

\section{Visión compartida}

La visión compartida se materializa en el deseo de la empresa por "incrementar la excelencia del negocio y calidad general que lleva a la satisfacción del cliente" (Wang y Wei, 2005, p. 1164). Este valor implica la ardua tarea de impulsar entre los miembros de la organización el propósito, las ganas de empezar el proceso de aprendizaje y continuarlo hasta obtener los resultados deseados. Mientras que el compromiso y la mentalidad abierta afectan la intensidad del aprendizaje, la visión compartida tiene más que ver con la dirección de este, de manera que todos los miembros de la organización sepan el propósito de este proceso para que los recursos sean aprovechados de forma eficiente (Sinkula et al., 1997).

\section{Mentalidad abierta}

Irónicamente, para lograr el aprendizaje es necesario "des-aprender", pues esto permite cuestionar los procesos utilizados, las teorías en uso, las viejas suposiciones, y las creencias que pueden ya no ser efectivas; estar dispuesto a hacerlo conlleva mantener una mente abierta al cambio y a nuevas posibilidades (Sinkula et al., 1997). Según Wang y Wei (2005), la mentalidad abierta "requiere de la autonomía y la flexibilidad en el empleo para producir ideas revolucionarias. El empoderamiento es crucial para formar tal ambiente de trabajo que conduzca al descubrimiento del dominio de nuevos productos o experimentar con diseños de procesos inusuales." (p. 1164). Los rápidos cambios tecnológicos son una buena razón para que los individuos en una organización desarrollen la madurez necesaria que les permita evaluar y criticar las rutinas acostumbradas y aceptar nuevas ideas (Sinkula et al., 1997; Calantone et al., 2002).

\section{Conocimiento intra-organizacional compartido}

El conocimiento intra-organizacional compartido se refiere al "conjunto de creencias o rutinas de comportamiento relacionadas con la difusión del aprendizaje entre las diferentes unidades de la organización" (Keskin, 2006, p. 404). El conocimiento se acumula dentro de cada individuo y al compartirlo se genera el aprendizaje organizacional. Para mantenerlo y no perderlo es necesario que se transmita, debido a los cambios de personal, y se almacene como una memoria de la empresa que pueda ser consultada antes de emprender futuras acciones (Calantone et al., 2002).

\section{Orientación al aprendizaje y desempeño empresarial}

En la literatura destaca el tema del desempeño de las empresas que promueven el aprendizaje organizacional. DiBella et al. (1996) conceptualizan la orientación al aprendizaje como la capacidad o proceso de una organización para mantener o mejorar su desempeño basándose en la experiencia, e involucrando la adquisición, diseminación y utilización de conocimiento. Dicho conocimiento podría ser potencialmente el recurso más productivo de una organización y factor indispensable para lograr una ventaja competitiva sostenible (Barney, 1991; Day, 1994; Slater y Narver, 1995; Grant, 1996; Sinkula et al., 1997; Hult, Snow y Kandemir, 2003; Wang y Wei, 2005). Esto se debe a que una organización orientada al aprendizaje procesa adecuadamente la información que recibe de sus clientes, competidores y canales de distribución, creando una 
competencia crítica para mejorar el desempeño y gracias a la información recabada, es capaz de prever cambios en el ambiente y el mercado logrando hacer a tiempo los ajustes necesarios (Calantone et al., 2002).

A partir de lo expuesto, se plantea la primera hipótesis:

H1: La orientación al aprendizaje de una empresa influye positivamente en su desempeño.

\section{Orientación al aprendizaje y enfoque a la innovación}

De la mano de la relación entre el aprendizaje y la generación de nuevo conocimiento, el enfoque a la innovación se conceptualiza como la medida en que una organización muestra disposición a aceptar nuevos conceptos cambiando los esquemas que utiliza (Hurley y Hult, 1998). Al ser llamado enfoque a la innovación se refiere también al conjunto de valores y creencias que estimulan la creatividad (Walter, Auer y Ritter, 2006), la invención (Hult et al., 2003), y la experimentación, promoviendo y apoyando nuevas ideas y la apertura hacia ellas (Lumpkin y Dess, 2001; Keskin, 2006), en muchas ocasiones asistido por la utilización de nuevos recursos tecnológicos, o resultando en nuevos productos, servicios o procesos (Lumpkin y Dess, 2001).

La orientación al aprendizaje representa el deseo de la organización por obtener conocimiento, y el enfoque a la innovación pone el acento en la búsqueda de cambio a través del conocimiento nuevo. Ambas capacidades están relacionadas porque una organización comprometida con el aprendizaje mejora su potencial innovador ya que es más probable que también esté comprometida con la innovación, invierta en tecnología de vanguardia, y la utilice para crear $\mathrm{y}$ vender nuevos productos. A su vez, las organizaciones que aprenden poseen conocimientos y habilidades para entender y anticipar las necesidades de los consumidores y también suelen desarrollar mayor capacidad innovadora que sus competidores porque monitorean y aprenden de sus acciones en el mercado (Damanpour, 1991; Cahill, et al., 1996; Calantone et al., 2002).

Slater y Narver (1995) sugieren que el principal enlace entre la innovación y el aprendizaje, se debe a que los valores que fomentan la orientación al aprendizaje crean en la empresa una tendencia a internarse en un proceso de búsqueda de conocimiento y fomentan la inquietud por romper el status quo de la compañía para conseguir un mejor desempeño, impulsando la exploración de nuevos mercados y el desarrollo de nuevos productos, servicios y tecnologías.

Derivado de lo anterior, se propone la siguiente hipótesis:

H2: La orientación al aprendizaje influye positivamente en el enfoque a la innovación de las empresas.

\section{Diferencias asociadas al tamaño y la edad de la empresa}

En los trabajos empíricos existe interés en analizar si la edad y tamaño de la organización influyen en la relación del aprendizaje con la innovación. En la mayoría de los casos, se observa que sí hay diferencias pero no se puede generalizar una conclusión al respecto, porque esta depende también de otros factores, como pueden ser el orden de entrada de las empresas al mercado, el sector económico, la diversidad en el portafolio de proyectos de investigación y desarrollo, el grado de competencia en el mercado, la presencia de economías de escala, el ciclo de vida del mercado, el grado de desarrollo tecnológico, las tasas de aprendizaje, o la red de contactos (Balasubramanian y Lee, 2008; Bianchini, Krafft, Quatraro y Ravix, 2015; Ciriaci, Moncada-Paternò-Castelo y Voigt, 2012; Coad, Segarra y Teruel, 2016; Hansen, 1992; Rogers, 2004; Hui, Radzi, Jenatabadi, Kasim y Radu, 2013; Prajogo, McDermott y McDermott, 2013; 
Sirén, Hakala, Wincent y Grichnik, 2017; Swee Lin Tan, Smyrnios y Xiong, 2014; Torres y Jasso, 2009).

En este contexto, este trabajo explora las siguientes hipótesis.

H3: Existen diferencias significativas en el enfoque a la innovación derivadas del tamaño de la empresa.

H4: Existen diferencias significativas en el enfoque a la innovación derivadas de la edad de la empresa.

En la figura 1 se dibuja el modelo que representa las relaciones planteadas en las hipótesis, así como las cuatro dimensiones que componen la orientación al aprendizaje.

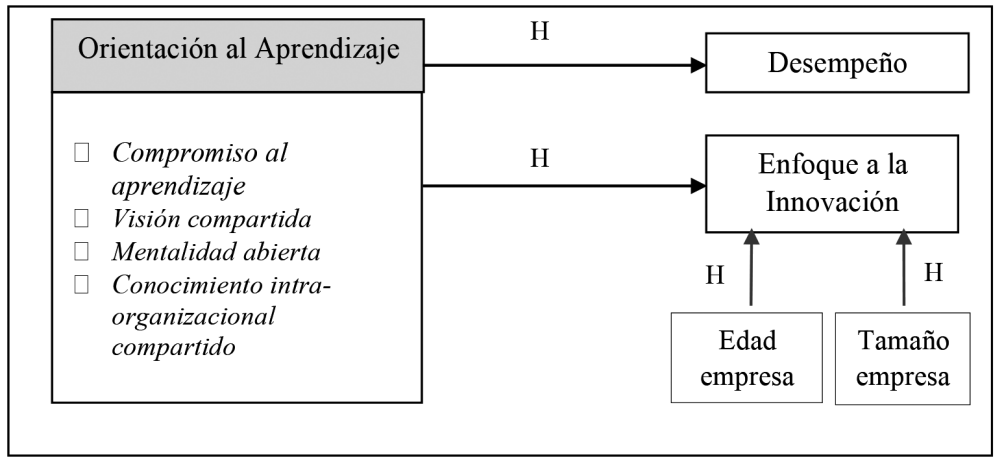

Figura 1. Modelo hipotético Fuente: Elaboración propia.

\section{Método}

\section{Recolección de datos}

En esta investigación se consideró como población objeto de estudio las micro, pequeñas y medianas empresas (Mipymes), y en concreto se desarrolló el trabajo empírico en empresas ubicadas en la ciudad de San Luis Potosí (México), pertenecientes a los sectores industrial, comercio y servicios, con hasta 100 empleados en aquellas de servicios o comercio y no más de 250 en la industria. Para obtener información sobre la población se consultó el Directorio Estadístico Nacional de Unidades Económicas (DENUE), el cual aglutina las empresas que se incluyeron en los Censos Económicos (Instituto Nacional de Estadística y Geografía [INEGI], 2009).

El muestreo fue aleatorio simple, y el tamaño muestral de 253 empresas con un intervalo de confianza del $90 \%$ y precisión del $5.1 \%$. Se diseñó un estudio cuantitativo y transversal, en el que se recogieron los datos durante los meses de septiembre, octubre y noviembre de 2012. Los informantes clave fueron los emprendedores o, en su caso, la persona que presidía la empresa, y el cuestionario fue aplicado cara a cara con el fin de obtener un número grande de respuestas, aclarar las posibles dudas y evitar respuestas erróneas debidas a mala interpretación.

El total de empresas que participaron en el estudio fue de 253: de las cuales, el 14.2\% pertenecía al sector industrial — principalmente a la industria manufacturera y de la construcción-, el 
$24.1 \%$ al comercio, y el $61.7 \%$ al sector de servicios —en orden de importancia, restaurantes y servicios de alojamiento, servicios de educación, servicios diversos, servicios profesionales, transportes, comunicaciones y servicios médicos-. Por el número de trabajadores, el 15\% de las empresas de la muestra eran micro, el $62 \%$ pequeñas y el $23 \%$ fueron medianas. En cuanto a la edad, el $37.5 \%$ tenía hasta 15 años en el mercado, el $42.7 \%$ entre 16 y 30 años, y el resto (19.8\%) más de 30 años.

\section{Variables}

El alcance de la investigación es descriptivo y causal. Las variables incluidas en el modelo y en el cuestionario son tres. Para medir la orientación al aprendizaje se adaptó el cuestionario de Calantone et al. (2002). En el caso del enfoque a la innovación, se utilizaron dos ítems basados en Covin y Slevin (1989). La sección del desempeño en el cuestionario incluyó diez ítems los cuales buscan determinar en qué medida el desempeño de la empresa es satisfactorio y fueron adaptados de Gupta y Govindarajan (1984); estos autores, en su estudio de pequeñas empresas, eligieron medidas subjetivas del desempeño por la falta de disposición de este sector empresarial a divulgar información financiera. En la tabla 1 se identifican las variables latentes, orientación al aprendizaje y sus dimensiones, enfoque a la innovación, y desempeño. Las variables observadas son los ítems que fueron codificados para una mejor identificación.

Tabla 1

Operacionalización de las variables

\begin{tabular}{|c|c|c|c|}
\hline \multirow{2}{*}{$\begin{array}{l}\text { Variable latente } \\
\quad \text { (Fuente) }\end{array}$} & \multirow{2}{*}{$\begin{array}{l}\text { Variable latente } \\
\text { (dimensiones) }\end{array}$} & \multicolumn{2}{|r|}{ Variables observadas } \\
\hline & & Código & Ítem \\
\hline \multirow{12}{*}{$\begin{array}{l}\text { Orientación al } \\
\text { aprendizaje } \\
\text { (Adaptado de } \\
\text { Calantone et al., } \\
\text { 2002) }\end{array}$} & \multirow[t]{4}{*}{$\begin{array}{l}\text { Compromiso al } \\
\text { aprendizaje }\end{array}$} & OA1 & $\begin{array}{l}\text { Los gerentes básicamente concuerdan en que la } \\
\text { habilidad de nuestra organización para aprender } \\
\text { es la clave de nuestra ventaja competitiva. }\end{array}$ \\
\hline & & $\mathrm{OA} 2$ & $\begin{array}{l}\text { Los valores básicos de esta organización incluyen } \\
\text { el aprendizaje como una clave para mejorar. }\end{array}$ \\
\hline & & OA3 & $\begin{array}{l}\text { El sentir en esta empresa es que el aprendizaje del } \\
\text { empleado es una inversión no un gasto. }\end{array}$ \\
\hline & & OA4 & $\begin{array}{c}\text { El aprendizaje en mi organización es visto como } \\
\text { un elemento necesario para garantizar la } \\
\text { sobrevivencia de la organización. }\end{array}$ \\
\hline & \multirow{5}{*}{ Visión compartida } & OA5 & Hay un propósito común en mi organización. \\
\hline & & OA6 & $\begin{array}{l}\text { Hay un acuerdo total de nuestra visión organiza- } \\
\text { cional a través de todos los niveles, funciones y } \\
\text { divisiones. }\end{array}$ \\
\hline & & OA7 & $\begin{array}{l}\text { Todos los empleados están comprometidos a las } \\
\text { metas de esta organización. }\end{array}$ \\
\hline & & OA8 & $\begin{array}{l}\text { Nuestros empleados se ven a sí mismos como } \\
\text { socios en la planeación de la dirección de la } \\
\text { organización. }\end{array}$ \\
\hline & & OA9 & $\begin{array}{l}\text { No tenemos miedo de reflexionar críticamente } \\
\text { sobre los supuestos compartidos que hemos } \\
\text { hecho de nuestros clientes. }\end{array}$ \\
\hline & \multirow[t]{2}{*}{ Mentalidad abierta } & OA10 & $\begin{array}{l}\text { El personal en esta empresa se da cuenta de que } \\
\text { la forma en la que perciben el mercado debe ser } \\
\text { continuamente cuestionada. }\end{array}$ \\
\hline & & OA11 & $\begin{array}{l}\text { Continuamente juzgamos la calidad de nuestras } \\
\text { decisiones y actividades realizadas en el tiempo. }\end{array}$ \\
\hline & $\begin{array}{l}\text { Conocimiento } \\
\text { intra-organizacional } \\
\text { compartido }\end{array}$ & OA12 & $\begin{array}{c}\text { Hay una buena parte de las conversaciones } \\
\text { organizacionales que mantienen vivas las } \\
\text { lecciones aprendidas. }\end{array}$ \\
\hline
\end{tabular}




\begin{tabular}{|c|c|c|c|}
\hline \multirow{5}{*}{$\begin{array}{l}\text { Enfoque a la } \\
\text { innovación } \\
\text { (Basado en } \\
\text { Covin y Slevin, } \\
\text { 1989) }\end{array}$} & & OA13 & $\begin{array}{l}\text { Siempre analizamos los esfuerzos organizaciona- } \\
\text { les que no tienen éxito y comunicamos amplia- } \\
\text { mente las lecciones aprendidas. }\end{array}$ \\
\hline & & OA14 & $\begin{array}{c}\text { Tenemos mecanismos específicos para compartir } \\
\text { las lecciones aprendidas en actividades organiza- } \\
\text { cionales, de un departamento a otro (de unidad a } \\
\text { unidad, de equipo a equipo). }\end{array}$ \\
\hline & & OA15 & $\begin{array}{l}\text { La alta gerencia enfatiza la importancia de com- } \\
\text { partir el conocimiento en nuestra compañía. }\end{array}$ \\
\hline & \multirow[t]{2}{*}{ No aplica } & I1 & $\begin{array}{l}\text { En general, la gerencia de mi empresa favorece } \\
\text { un fuerte énfasis en investigación y desarrollo, } \\
\text { liderazgo tecnológico e innovaciones. }\end{array}$ \\
\hline & & I2 & $\begin{array}{c}\text { Mi empresa ha lanzado al mercado muchas } \\
\text { nuevas líneas de productos o servicios en los } \\
\text { últimos cinco años. }\end{array}$ \\
\hline \multirow[t]{2}{*}{ Desempeño } & \multirow[t]{10}{*}{ No aplica } & D1 & Nivel de ventas. \\
\hline & & D2 & Tasa de crecimiento de ventas. \\
\hline \multirow{8}{*}{$\begin{array}{l}\text { (Adaptado de } \\
\text { Gupta y Govin- } \\
\text { darajan, 1984) }\end{array}$} & & D3 & Flujo de efectivo. \\
\hline & & D4 & Rendimiento del capital de los socios. \\
\hline & & D5 & $\begin{array}{l}\text { Porcentaje o margen de utilidad bruta } \\
\text { (utilidad bruta/ventas). }\end{array}$ \\
\hline & & D6 & $\begin{array}{l}\text { Utilidades netas de las operaciones (ganancias } \\
\text { antes de intereses e impuestos). }\end{array}$ \\
\hline & & D7 & Volumen de activo (tamaño de la inversión). \\
\hline & & D8 & Crecimiento en el número de empleados. \\
\hline & & D9 & Rendimiento sobre la inversión \\
\hline & & D10 & $\begin{array}{l}\text { Capacidad de financiar el crecimiento de la } \\
\text { empresa a partir de utilidades obtenidas. }\end{array}$ \\
\hline
\end{tabular}

Fuente: elaboración propia.

Los ítems de las tres variables fueron calificados en una escala de Likert para su evaluación, que va desde 1 (totalmente en desacuerdo o nada satisfecho) a 7 (totalmente de acuerdo o muy satisfecho). El tamaño y edad de las empresas se midieron, respectivamente, por el número de empleados y de años en el mercado al momento de aplicación del cuestionario. Los datos recogidos se analizaron utilizando la aplicación estadística SPSS versión 20 y Amos versión 20.

Como se observa en la tabla 2, las medidas utilizadas para establecer validez convergente y discriminante de las escalas fueron: fiabilidad compuesta (FC) y varianza extraída promedio (VEP). La fiabilidad compuesta de las variables está muy por encima del nivel recomendado de 0.7. La varianza extraída de las tres variables supera el nivel recomendado del 0.5; esto es, más de la mitad de la varianza de los indicadores se tiene en cuenta para el factor. Por último, se encontró que la raíz cuadrada de la varianza extraída (señalada en negritas) es mayor que las correlaciones de los constructos y dimensiones de orientación al aprendizaje, lo cual indica que hay validez discriminante.

Tabla 2

Validez convergente y discriminante de las escalas

\begin{tabular}{lcccccc}
\hline & CR & AVE & 1 & 2 & 3 & 4 \\
\hline 1 Compromiso al aprendizaje & 0.894 & 0.738 & 0.859 & & & \\
2 Conocimiento organizacional compartido & 0.891 & 0.672 & 0.798 & 0.819 & & \\
3 Enfoque a la innovación & 0.712 & 0.562 & 0.448 & 0.460 & 0.750 & \\
4 Desempeño & 0.914 & 0.642 & 0.246 & 0.241 & 0.189 & 0.801 \\
\hline
\end{tabular}

Fuente: Elaboración propia 


\section{Análisis de resultados}

\section{Descripción de la muestra}

Los dueños o directores de empresa fueron los informantes clave en la encuesta y su perfil es el siguiente. El $43.1 \%$ de las empresas de la muestra pertenece a mujeres y el $56.9 \%$ a hombres. Su edad promedio es de 43 años, siendo el mínimo 19 y el máximo 74 años. El 67.2\% de los encuestados tiene una carrera profesional concluida, el 13\% cuenta con estudios de posgrado y sólo el $18.6 \%$ tiene carrera profesional no concluida o un nivel de estudio menor, siendo primaria el más bajo (ver tabla 3 ).

Con respecto a las organizaciones objeto de estudio, se tiene que el valor promedio de edad de la empresa es de 22.3 años - hay empresas que no han cumplido un año de haberse fundado y el máximo es de 155 años- El sector industrial está representado por un $14.2 \%$, comercio con un $24.1 \%$ y servicios con un $61.7 \%$. El $15 \%$ son micro empresas, el $62 \%$ son pequeñas y el $23 \%$ son medianas.

Tabla 3

Caracterización de la muestra

\begin{tabular}{ll}
\hline Dueños o directores de la empresa & Empresas \\
\hline Sexo: & Edad: \\
$56.9 \%$ hombres & Desde 0 años \\
$43.1 \%$ mujeres & Hasta 155 años \\
& Promedio: 22.3 años \\
\hline Edad: & Sector: \\
Mínimo de edad 19 años & Servicios 61.7\% \\
Máximo de edad 74 años & Comercio 24.1\% \\
\hline Promedio: 43 años & Industrial 14.2\% \\
Nivel de estudio concluido: & Tamaño: \\
$14.2 \%$ posgrado & Micro 15\% \\
$67.2 \%$ carrera profesional & Pequeñas 62\% \\
$15.4 \%$ preparatoria o carrera técnica & Medianas 23\% \\
$3.2 \%$ secundaria o primaria & \\
\hline
\end{tabular}

Tamaño de empresa: Micro: hasta 10 empleados. Pequeña: hasta 30 (comercio) y 50 empleados (industria y servicios). Mediana: hasta 100 (comercio y servicios) y 250 (industria).

Fuente: elaboración propia.

\section{Modelo de medida y análisis descriptivo de las escalas}

Los resultados del modelo de medida, en el que se utilizó el método de máxima verosimilitud, indican que el modelo ajustó bien al realizar la prueba de chi cuadrada, $\mathrm{X}^{2}(\mathrm{~N}=253, \mathrm{df}=84)$ $=132.724, \mathrm{p}<.001$.

Para los constructos de orientación al aprendizaje, enfoque a la innovación y desempeño, los ítems y las cargas estandarizadas se especifican en la tabla 4 . Podemos observar que todas ellas son significativas al nivel $\mathrm{p}<.001$, y superiores a 0.5 , lo que indica que las variables latentes están significativamente representadas por sus respectivas variables observadas. Del constructo orientación al aprendizaje los ítems OA1, OA5, OA6, OA7, OA8, OA9, OA10 y OA11 fueron eliminados por tener cargas estandarizadas menores a 0.5. y en algunos casos por ser redundantes — por lo que quedaron únicamente representadas las dimensiones de compromiso al aprendizaje y conocimiento intra-organizacional compartido-. Por las mismas razones, en el caso del desempeño también se eliminaron los ítems D1, D5, D7 y D9. 
En la escala de OA, se observa que las medias más altas y superiores a 6 con una interpretación "muy de acuerdo" son para los ítems OA4 y OA3, que se refieren a que "El aprendizaje en su organización es visto como un elemento necesario para garantizar la sobrevivencia de la organización” y "El sentir en la empresa es que el aprendizaje del empleado es una inversión no un gasto". Así como OA15 y OA2 que mencionan que "Los valores básicos de la organización incluyen el aprendizaje como una clave para mejorar" y "La alta gerencia enfatiza la importancia de compartir el conocimiento en nuestra compañía". El resto de los ítems de OA se ubican en una media que se interpreta como "de acuerdo". Esto refleja que las empresas dicen estar comprometidas con el aprendizaje y lo tienen claro en el discurso pero el consenso disminuye cuando valoran prácticas específicas de desarrollo de capacidades colectivas de conocimiento que son esenciales para la supervivencia organizacional.

En la variable innovación la media disminuye, siendo la más alta de 5.091 — que se interpreta como "de acuerdo" - para el ítem "En general, la gerencia de mi empresa favorece un fuerte énfasis en investigación y desarrollo, liderazgo tecnológico e innovaciones”. Por otro lado, el ítem "La empresa ha lanzado al mercado muchas nuevas líneas de productos o servicios en los últimos cinco años" alcanza una media interpretada como "ni de acuerdo, ni en desacuerdo". De nueva cuenta, se observa que, frente al discurso, el consenso disminuye cuando se habla de la implementación. Además, la innovación alcanza medias inferiores que el aprendizaje; esto puede explicarse por la mayor complejidad que implica innovar, lo cual requiere y produce aprendizaje, pero la relación inversa no siempre es cierta.

En cuanto al desempeño, se muestra que, a excepción de un ítem (D4), todos tienen una media mayor a 5 interpretada como "satisfecho" con el criterio mencionado. El indicador con la media más baja es el que se refiere al rendimiento del capital de los socios. La razón podría encontrarse en que los informantes clave eran dueños o socios, por lo que esta pregunta tiene connotaciones más subjetivas para ellos.

Tabla 4

Valores estimados del modelo de medida y análisis descriptivo de los ítems

\begin{tabular}{cccccccccl}
\hline & Ruta & \multicolumn{9}{c}{$\begin{array}{c}\text { Carga } \\
\text { estandarizada }\end{array}$} & Valor t & Sig. & Mín & Máx & Media & Interpretación \\
\hline OA2 & $<---$ & CL & 0.839 & $*$ & $*$ & 1 & 7 & 6.281 & Muy de acuerdo \\
OA3 & $<---$ & CL & 0.897 & 17.395 & $\mathrm{p}<.001$ & 2 & 7 & 6.383 & Muy de acuerdo \\
OA4 & $<---$ & CL & 0.840 & 15.983 & $\mathrm{p}<.001$ & 2 & 7 & 6.399 & Muy de acuerdo \\
OA12 & $<---$ & IKS & 0.821 & $*$ & $*$ & 2 & 7 & 5.945 & De acuerdo \\
OA13 & $<---$ & IKS & 0.873 & 16.229 & $\mathrm{p}<.001$ & 1 & 7 & 5.874 & De acuerdo \\
OA14 & $<---$ & IKS & 0.777 & 13.846 & $\mathrm{p}<.001$ & 1 & 7 & 5.609 & De acuerdo \\
OA15 & & IKS & 0.804 & 14.520 & $\mathrm{p}<.001$ & 3 & 7 & 6.287 & Muy de acuerdo \\
I1 & $<---$ & Inn & 0.880 & $*$ & $*$ & 1 & 7 & 5.091 & De acuerdo \\
I2 & $<---$ & Inn & 0.591 & 5.084 & $\mathrm{p}<.001$ & 1 & 7 & 4.812 & Ni de acuerdo ni en desacuerdo \\
D2 & $<---$ & Des & 0.778 & 14.238 & $\mathrm{p}<.001$ & 1 & 7 & 5.292 & Satisfecho \\
D3 & $<---$ & Des & 0.876 & 16.970 & $\mathrm{p}<.001$ & 1 & 7 & 5.128 & Satisfecho \\
D4 & $<---$ & Des & 0.771 & 14.054 & $\mathrm{p}<.001$ & 1 & 7 & 4.972 & Relativamente satisfecho \\
D6 & $<---$ & Des & 0.893 & 17.477 & $\mathrm{p}<.001$ & 1 & 7 & 5.045 & Satisfecho \\
D8 & $<---$ & Des & 0.641 & 10.996 & $\mathrm{p}<.001$ & 1 & 7 & 5.230 & Satisfecho \\
D10 & $<---$ & Des & 0.824 & $*$ & $*$ & 1 & 7 & 5.182 & Satisfecho \\
\hline \multicolumn{7}{c}{ Ajuste del modelo } \\
\hline
\end{tabular}

Fuente: elaboración propia.

Nota: *Valores no calculados porque la ponderación se fijó en 1.00 para fijar la varianza del constructo. 


\section{Modelo estructural}

Los resultados del modelo de ecuaciones estructurales, en el que se utilizó el método de máxima verosimilitud, indican que el modelo ajustó bien al realizar la prueba de chi cuadrada, $\mathrm{X}^{2}(\mathrm{~N}=253, \mathrm{df}=112)=159.149, \mathrm{p}<.002$.

Adicionalmente, en el caso de los índices de bondad de ajuste incremental (ver tabla 6), IFI, TLI y CFI están todos por encima de 0.9 en un rango de 0.976 a 0.980 lo cual se considera aceptable. Lo mismo sucede con el error cuadrático medio de aproximación (RMSEA), el cual tiene un valor de 0.041 y está en un nivel por debajo del máximo recomendado de 0.08 (Hair et al., 1999; Schumacker y Lomax, 2004; Pesämaa, Shoham, Wincent, y Ruvio, 2013). Asimismo, los resultados indican que los pesos de las regresiones estandarizadas son significativos por la prueba del valor $\mathrm{t}(>=1.96, \mathrm{p}<=.05)$.

Ya que se sugiere en la literatura que el tamaño y la edad de la empresa podrían tener impacto en la innovación, estas dos variables fueron probadas como variables de control. No se encontró efecto significativo por parte de la edad de la empresa; sin embargo, hubo efecto positivo significativo $(\mathrm{p}<0.05)$ por parte del tamaño de la empresa sobre el enfoque a la innovación y, tomando en cuenta el coeficiente estandarizado de regresión (0.182), podemos decir que el efecto es positivo débil (ver tabla 5).

Más allá de que la muestra tiene una gran dispersión en la edad de las empresas medida en años, la razón de lo anterior tiene que ver con que el aprovechamiento de factores externos y desarrollo de capacidades organizacionales — que facilitan la innovación—, son más factibles en las empresas de mayor tamaño y se explican menos por la permanencia en el mercado — el tiempo- (Torres y Jasso, 2009). El que la relación sea débil, podría deberse a que la variable no mantiene las dimensiones relativas a visión compartida y mentalidad abierta. Además, se intuye una falta de sistematización de las prácticas de aprendizaje, lo que menoscaba los resultados innovadores.

\begin{tabular}{cccccc}
\multicolumn{7}{c}{ Tabla 5} \\
Resultados del modelo estructural \\
\hline \multicolumn{5}{c}{ Carga } \\
\hline \multicolumn{7}{c}{ Ruta } & estandarizada & Valor t & Sig. \\
\hline Des & $<---$ & OA & 0.278 & 3.807 & $\mathrm{p}<.001$ \\
IKS & $<---$ & OA & 0.911 & 8.125 & $\mathrm{p}<.001$ \\
CL & $<---$ & OA & 0.876 & $*$ & $*$ \\
Inn & $<---$ & OA & 0.527 & 6.403 & $\mathrm{p}<.001$ \\
OA2 & $<---$ & OA & 0.840 & $*$ & $*$ \\
OA3 & $<---$ & OA & 0.896 & 17.382 & $\mathrm{p}<.001$ \\
OA4 & $<---$ & OA & 0.841 & 16.000 & $\mathrm{p}<.001$ \\
OA12 & $<---$ & OA & 0.820 & $*$ & $*$ \\
OA13 & $<---$ & OA & 0.873 & 16.218 & $\mathrm{p}<.001$ \\
OA14 & $<---$ & OA & 0.778 & 13.855 & $\mathrm{p}<.001$ \\
OA15 & $<---$ & OA & 0.804 & 14.516 & $\mathrm{p}<.001$ \\
I1 & $<---$ & Inn & 0.840 & $*$ & $*$ \\
I2 & $<---$ & Inn & 0.619 & 5.787 & $\mathrm{p}<.001$ \\
D2 & $<---$ & Des & 0.778 & 14.233 & $\mathrm{p}<.001$ \\
D3 & $<---$ & Des & 0.876 & 16.975 & $\mathrm{p}<.001$ \\
D4 & $<---$ & Des & 0.771 & 14.054 & $\mathrm{p}<.001$ \\
D6 & $<---$ & Des & 0.893 & 17.464 & $\mathrm{p}<.001$ \\
D8 & $<---$ & Des & 0.641 & 10.997 & $\mathrm{p}<.001$ \\
D10 & $<---$ & Des & 0.824 & $*$ & $*$ \\
Inn & $<---$ & Tamaño & 0.182 & 2.724 & $\mathrm{p}<.050$ \\
Inn & $<---$ & Edad & 0.042 & 0.635 & $\mathrm{n} . \mathrm{s}$ \\
\hline
\end{tabular}




Ajuste del modelo
\[ \begin{array}{l}\mathrm{X} 2=159.149 \mathrm{df}=112 \mathrm{p}=0.002 \\ \mathrm{CFI}=0.980\end{array} \]
RMSA $=0.041$
*Valores no calculados porque la ponderación se fijó
en 1.00 para fijar la varianza del constructo.
n.s.= no significativo
Fuente: elaboración propia.

Con base en el análisis anterior se obtuvo el modelo de orientación al aprendizaje, innovación y desempeño (ver figura 2). La orientación al aprendizaje explica un $8 \%$ de la varianza de la variable dependiente desempeño y un $31 \%$ del enfoque a la innovación. Esto tiene su razón en la directa relación que existe entre aprendizaje, conocimiento e innovación; mientras que en el desempeño influyen muchos otros factores internos y externos a la organización.

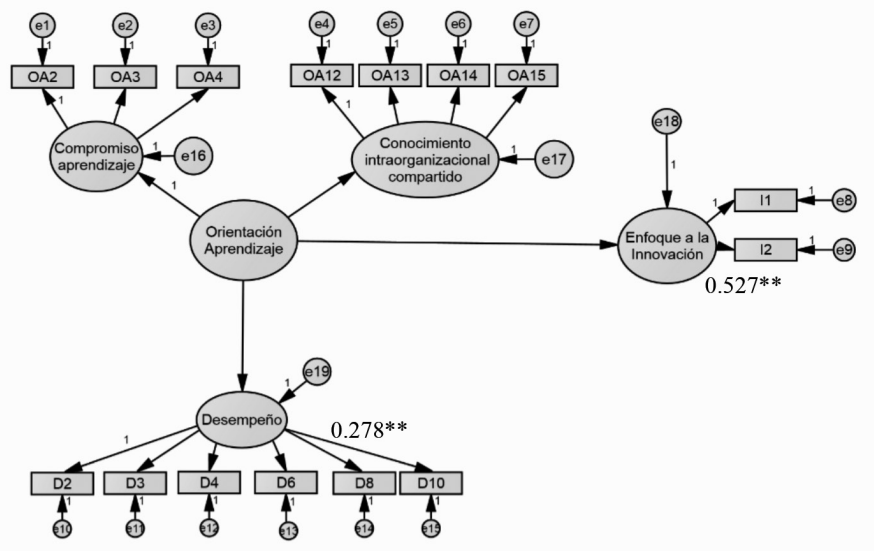

Figura 2. Resultados del modelo estructural de orientación al aprendizaje, innovación y desempeño. Nota: $* * *$ corresponde a $\mathrm{p}=<0.001 ; * *$ corresponde a $\mathrm{p}=<0.05$

Fuente: elaboración propia.

Finalmente, a modo de resumen, los hallazgos permiten afirmar que se encontró evidencia empírica a favor de la relación positiva entre orientación al aprendizaje y desempeño y también con la innovación; además, esta última se ve influenciada por el tamaño, pero no por la edad de la empresa (ver tabla 6).

Tabla 6

Resultados en relación con la hipótesis

\begin{tabular}{lc}
\hline Hipótesis & Hallazgos \\
\hline H1: La orientación al aprendizaje de una empresa influye positivamente en su desempeño. & Aceptada \\
H2: La orientación al aprendizaje influye positivamente en el enfoque a la innovación de & Aceptada \\
las empresas. & \\
H3: Existen diferencias significativas en el enfoque a la innovación derivadas del tamaño & Aceptada \\
de la empresa. & \\
H4: Existen diferencias significativas en el enfoque a la innovación derivadas de la edad & Rechazada \\
de la empresa. &
\end{tabular}




\section{Discusión y conclusiones}

Esta investigación se llevó a cabo en el contexto de una economía emergente, y con una muestra de empresas que incluye una mayor proporción del sector servicios (61.7\%), una elevada presencia de empresas que no tienen más de 50 trabajadores (77\%), y un promedio de edad de 22.3 años, lo que indica que no son empresas jóvenes, según los criterios utilizados en la literatura (Lin Tan et al., 2014; Coad et al., 2016; Sirén et al., 2017).

El principal resultado es que la orientación al aprendizaje explica un $8 \%$ de la varianza del desempeño y un $31 \%$ del enfoque a la innovación, lo cual está en línea con la literatura empírica que reporta efectos positivos del aprendizaje en innovación y resultados organizacionales (Calantone et al., 2002; Jiménez-Jiménez y Sanz-Valle, 2011; Alegre y Chiva 2013; Martínez Serna et al., 2016 y 2018). A la vista de los hallazgos, se concluye también, que el aprendizaje incide en mayor medida en el enfoque hacia la innovación que en el desempeño —en este sentido, Gomes y Wojahn (2017) encuentran que la capacidad de aprendizaje organizacional influye en la innovación, pero no en el desempeño-.

En este modelo no se debe ignorar que el tamaño de la empresa, medido en número de empleados, resulta significativo a un nivel $\mathrm{p}<.050$, lo que sugiere que a mayor número de empleados mayor enfoque a la innovación (Hui et al., 2013). Por su parte, el trabajo de Jiménez-Jiménez y Sanz-Valle (2011) reporta que el efecto positivo de la orientación al aprendizaje sobre la innovación y el desempeño es mayor en las empresas más pequeñas, y aduce que las grandes tienen más recursos para invertir en innovación y esto las hace menos dependientes del enfoque al aprendizaje a la hora de innovar. Sin embargo, consideramos que precisamente el hecho de que cuenten con más medios facilita su enfoque a la innovación, lo cual pone a las micro empresas en desventaja. En este sentido, Torres y Jasso (2009) concluyen que las carencias en el desarrollo de factores internos relacionados con la innovación — tales como la realización de actividades de capacitación, investigación y desarrollo - limitan la supervivencia y crecimiento de las PYMES.

Según Jasso (2004), la relación entre la trayectoria tecnológica de las empresas y su ciclo de vida pasa no solo por la tecnología y propone considerar el mercado y la producción, analizando las capacidades de gestión y de aprendizaje organizacional. La literatura afirma que el ciclo de vida sí afecta las capacidades empresariales (Bianchi et al., 2015; Coad et al., 2016; Sirén et al., 2017), pero en este trabajo no se han encontrado diferencias significativas en el enfoque a la innovación derivadas de la edad de la empresa. Este hecho merece un mayor análisis en futuras investigaciones, que pueden incluir otras variables del entorno de las empresas, para mejorar la comprensión del fenómeno del enfoque a la innovación.

Al analizar más en detalle la orientación al aprendizaje, observamos que el compromiso con el aprendizaje, unido a la tendencia a compartir el conocimiento dentro de la organización, fomentan las acciones de investigación y desarrollo, así como el lanzamiento al mercado de nuevos productos o servicios (carga estandarizada de la orientación al aprendizaje hacia el enfoque a la innovación 0.527). Sin embargo, quedan fuera las dimensiones relativas a la visión compartida y la mentalidad abierta. Esto refleja las debilidades en la dirección estratégica de estas empresas, que no comparten expectativas, y se resisten al cambio en un ecosistema poco innovador que tampoco fomenta la necesidad de cambio.

El presente estudio contribuye con la literatura aportando evidencia de una economía emergente y de empresas pequeñas que se han consolidado en sus respectivos mercados. Además, los resultados tienen implicaciones a nivel gerencial en el sentido de que estas empresas deben 
comprometerse con el aprendizaje si desean construir mejores capacidades (Torres, 2006) y ser más competitivas, pero tienen que revisar la forma en la que actualmente aprenden. En este sentido, es necesario que promuevan una visión más innovadora, e implementen acciones de desarrollo de capacidades internas relativas a la generación y aplicación de conocimiento y al aprendizaje organizacional.

En cuanto a las limitaciones del estudio, conviene señalar su diseño transeccional, por lo que hay que tener reservas a la hora de interpretar la causalidad en las relaciones planteadas. Además, en la práctica, el efecto del aprendizaje en la innovación y el desempeño suele observarse con retraso por lo que se sugiere la realización de estudios longitudinales.

\section{Agradecimientos}

Los autores agradecen a la Comisión de Investigación y Desarrollo Tecnológico CICTD, de la Universidad Autónoma de San Luis Potosí por su apoyo para la realización de esta investigación. Dicho apoyo fue otorgado dentro del marco de la convocatoria del Fondo de Apoyo a la Investigación FAI. Folio del proyecto C17-FAI-06-30.30.

\section{Referencias}

Alegre, J., \& Chiva, R. (2008). Assessing the impact of organizational learning capability on product innovation performance: An empirical test. Technovation, 28(6), 315-326. https://doi.org/10.1016/j.technovation.2007.09.003

Alegre, J., \& Chiva, R. (2013). Linking entrepreneurial orientation and firm performance: The role of organizational learning capability and innovation performance. Journal of Small Business Management, 51(4), 491-507. https:// doi.org/10.1111/jsbm.12005

Baker, W. E., \& Sinkula, J. M. (1999). The synergistic effect of market orientation and learning orientation on organizational performance. Journal of the Academy of Marketing Science, 27(4), 411-427. https://doi. org/10.1177/0092070399274002

Balasubramanian, N., \& Lee, J. (2008). Firm age and innovation. Industrial \& Corporate Change, 17(5), 1019-1047. https://doi.org/10.1093/icc/dtn028

Barney, J. (1991). Firm resources and sustained competitive advantage. Journal of Management, 17(1), 99-120. https:// doi.org/10.1177/014920639101700108

Bianchini, S., Krafft, J., Quatraro, F., \& Ravix, J. (2015). Corporate governance, innovation and firm age: Insights and new evidence. GREDEG Working Papers Series, 68(275), 297-326.

Cahill, D., Lumpkin, G. T., Dess, G. G., Johnson, P. C., Purser, R. E., Montuori, A., . . Abrahamson, E. (1996). Dialogue / enriching the entrepreneurial orientation Construct-A reply to "Entrepreneurial orientation or pioneer advantage". Academy of Management Review, 21(3), 603-618. https://doi.org/10.5465/AMR.1996.26115125

Calantone, R. J., Cavusgil, S. T., \& Zhao, Y. (2002). Learning orientation, firm innovation capability, and firm performance. Industrial Marketing Management, 31(6), 515-524. https://doi.org/10.1016/S0019-8501(01)00203-6

Cavallo, E. \& Powell, E. (coord.) (2018). La hora del crecimiento. Informe macroeconómico de América Latina y el Caribe. 2018. Washington, DC: Banco Interamericano de Desarrollo.

Ciriaci, D., Moncada-Paternò-Castello, P., \& Voigt, P. (2012). Does size or age of innovative firms affect their growth persistence? - evidence from a panel of innovative Spanish firms -. (No. EUR 25477 EN). Seville: Joint Research Centre. Disponible en: http://iri.jrc.ec.europa.eu/documents/10180/bf2da533-f98e-4c5d-9eb0-0d5caeedc9c6. Consultado: 02/09/2017.

Coad, A., Segarra, A., \& Teruel, M. (2016). Innovation and firm growth: Does firm age play a role? Research Policy, 45(2), 387-400. https://doi.org/10.1016/j.respol.2015.10.015

Covin, J. G., \& Slevin, D. P. (1989). Strategic management of small firms in hostile and benign environments. Strategic Management Journal, 10(1), 75-87. https://doi.org/10.1002/smj.4250100107 
Damanpour, F. (1991). Organizational innovation: A meta-analysis of effects of determinants and moderators. Academy of Management Journal, 34(3), 555-590. https://doi.org/10.2307/256406

Day, G.S. (1994). The capabilities of market-driven organizations. Journal of Marketing, 58(4), 37-52. https://doi. org/10.2307/1251915

Dibella, A. J., Nevis, E. C., \& Gould, J. M. (1996). Understanding organizational learning capability. Journal of Management Studies, 33(3), 361-379. https://doi.org/10.1111/j.1467-6486.1996.tb00806.x

Dutrénit, G. (2000). Learning and knowledge management in the firm: From knowledge accumulation to strategic capabilities. Massachusetts: Edward Elgar Publishing.

Estrada, S., \& Dutrénit, G. (2007). Gestión del conocimiento en pymes y desempeño competitivo. Engevista, 9(2), 129-148. https://doi.org/10.22409/engevista.v9i2.203

Fraj, E., Matute, J., \& Melero, I. (2013). El aprendizaje y la innovación como determinantes del desarrollo de una capacidad de gestión medioambiental proactiva. Cuadernos de Economía y Dirección de la Empresa, 16(3), 180193. https://doi.org/10.1016/j.cede.2012.10.001

Gomes, G. \& Wojahn, R. M. (2017). Organizational learning capability, innovation and performance: study in small and medium-sized enterprises (SMES). Revista de Administração, 52, 163-175. https://doi.org/10.1016/j. rausp.2016.12.003

Grant, R. M. (1996). Toward a knowledge-based theory of the firm. Strategic Management Journal, 17(S2), 109-122. https://doi.org/10.1002/smj.4250171110

Gupta, A. K. \& Govindarajan, V. (1984). Business unit strategy, managerial characteristics, and business unit effectiveness at strategy implementation. Academy of Management Journal, 27(1), 25-41. https://doi.org/ 10.2307/255955

Hair, J., Anderson, R., Tatham, R., \& Black, W. (1999). Análisis multivariante (5th ed.). Madrid: Pearson. Prentice Hall.

Hansen, J. A. (1992). Innovation, firm size, and firm age. Small Business Economics, 4(1), 37-44. https://doi. org/10.1007/BF00402214

Huber, G. P. (1991). Organizational learning: The contributing processes and the literatures. Organization Science, 2(1), 88-115. https://doi.org/10.1287/orsc.2.1.88

Hui, H., Radzi, W. M., Jasimah, C. W., Jenatabadi, H. S., Abu Kasim, F., \& Radu, S. (2013). The impact of firm age and size on the relationship among organizational innovation, learning, and performance: A moderation analysis in Asian food manufacturing companies. Interdisciplinary Journal of Contemporary Research in Business, 5(4), 166-174.

Hult, G. T., Snow, C. C., \& Kandemir, D. (2003). The role of entrepreneurship in building cultural competitiveness in different organizational types. Journal of Management, 29(3), 401-426. https://doi.org/10.1016/S01492063_03_00017-5

Hurley, R. F., \& Hult, G. T. M. (1998). Innovation, market orientation, and organizational learning: An integration and empirical examination. Journal of Marketing, 62(3), 42-54. https://doi.org/10.2307/1251742

Instituto Nacional de Estadística y Geografía [INEGI] (2009). Censos Económicos 2009. México: INEGI. Disponible en: http://www.inegi.org.mx/est/contenidos/espanol/proyectos/censos/ce2009/. Consultado: 20/06/2012.

Jasso, J. (2004). Trayectoria tecnológica y ciclo de vida de las empresas: una interpretación metodológica acerca del rumbo de la innovación. Contaduría y Administración, 214, 83-96. https://doi.org/10.22201/fca.24488410e.2004.469

Jiménez-Jiménez, D., \& Sanz-Valle, R. (2011). Innovation, organizational learning, and performance. Journal of Business Research, 64(4), 408-417. https://doi.org/10.1016/j.jbusres.2010.09.010

Keskin, H. (2006). Market orientation, learning orientation, and innovation capabilities in SMEs: An extended model. European Journal of Innovation Management, 9(4), 396-417. https://doi.org/10.1108/14601060610707849

Liu, C-H., (2017). Creating competitive advantage: Linking perspectives of organization learning, innovation behavior and intellectual capital. International Journal of Hospitality Management 66, 13-23. http://dx.doi.org/10.1016/j. ijhm.2017.06.013

Liu, S. S., Luo, X., \& Shi, Y. (2002). Integrating customer orientation, corporate entrepreneurship, and learning orientation in organizations-in-transition: An empirical study. International Journal of Research in Marketing, 19(4), 367-382. https://doi.org/10.1016/S0167-8116(02)00098-8 
Lumpkin, G. T., \& Dess, G. G. (2001). Linking two dimensions of entrepreneurial orientation to firm performance: The moderating role of environment and industry life cycle. Journal of Business Venturing, 16(5), 429-451. https://doi. org/10.1016/S0883-9026(00)00048-3

Martínez Serna, M. C., Vega Martínez, J. E. \& Vega Martínez, J. (2016). The impact of learning orientation on innovation and performance in SME's in México. International Review of Management and Business Research, 5(1), 48-64.

Martínez Serna, M. C., Vega Martínez, J. E. \& Eternod Domenech, V. (2018). The influence of organizational commitment, learning orientation on innovation of Mexican SMEs. Contaduría y Administración, 63(3), 1-19. https:// doi.org/10.22201/fca.24488410e.2018.1411

Mendoza, J. G. \& Valenzuela, A. (2014). Aprendizaje, innovación y gestión tecnológica en la pequeña empresa. Un estudio de las industrias metalmecánica y de tecnologías de información en Sonora. Contaduría y Administración, 59(4), 253-284. https://doi.org/10.1016/S0186-1042(14)70162-7

Ndiaye, N., Razak, L. A., Nagayev, R., \& Ng, A. (2018). Demystifying small and medium enterprises' (SMEs) performance in emerging and developing economies. Borsa Istanbul Review. Publicación anticipada en línea. https:// doi.org/10.1016/j.bir.2018.04.003

OECD (2017). OECD Compendium of Productivity Indicators 2017. Paris: OECD Publishing. https://doi.org/10.1787/ pdtvy-2017-en

OECD (2018). Main Science and Technology Indicators. MSTI 2017-2 / Release date: 1 March 2018. Disponible en http://www.oecd.org/sti/msti.htm. Consultado: 15/05/2018.

Pastor, M. P. (2012). Innovación en la industria mexicana: reflexiones a partir del estudio del sistema de innovación en San Luis Potosí. Revista Legislativa de Estudios Sociales y de Opinión Pública, 5(10), 121-145.

Pastor, M. P., Rodríguez, P. I., \& Ramos, A. E. (2017). Efectos del financiamiento público a la innovación: perspectiva microeconómica a partir de un estudio en pequeñas empresas. Región y sociedad, 29(70), 203-229. https://doi. org/10.22198/rys.2017.70.a346

Pesämaa, O., Shoham, A., Wincent, J., \& Ruvio, A. A. (2013). How a learning orientation affects drivers of innovativeness and performance in service delivery. Journal of Engineering and Technology Management, 30(2), 169-187. https://doi.org/10.1016/j.jengtecman.2013.01.004

Pezeshkan, A., Fainshmidt, S., Nair, A., Lance Frazier, M., \& Markowski, E. (2016). An empirical assessment of the dynamic capabilities-performance relationship. Journal of Business Research, 69(8), 2950-2956. https://doi. org/10.1016/j.jbusres.2015.10.152

Prajogo, D. I., McDermott, C. M., \& McDermott, M. A. (2013). Innovation orientations and their effects on business performance: contrasting small- and medium-sized service firms. $R \& D$ Management, 43(5), 486-500. https://doi. org/10.1111/radm.12027

Rhee, J., Park, T., \& Lee, D. H. (2010). Drivers of innovativeness and performance for innovative SMEs in South Korea: Mediation of learning orientation. Technovation, 30, 65-75. https://doi.org/10.1016/j.technovation.2009.04.008

Rodríguez, P., Fuentes M. M., \& Rodríguez, L. (2014). Strategic Capabilities and Performance in WomenఐOwned Businesses in Mexico. Journal of Small Business Management 52(3), 541-554. https://doi.org/10.1111/jsbm.12048.

Rodríguez, P. I., Ramos, A. E., \& Pastor, M. P. (2015). La orientación al mercado en las mipymes de San Luis Potosí. Nova Scientia, 7(15), 436-461. https://doi.org/10.21640/ns.v7i15.141.

Rogers, M. (2004). Networks, firm size and innovation. Small Business Economics, 22(2), 141-153. https://doi. org/10.1023/B:SBEJ.0000014451.99047.69

Schumacker, R. E., \& Lomax, R. G. (2004). A beginner's guide to structural equation modeling. New Jersey: Lawrence Erlbaum Associates.

Segura, F. J., Borjas, H. A., \& Sifuentes, A. J. (2014). Evaluación del ambiente innovador en San Luis Potosí. Región y sociedad 26(61), 161-200.

Senge, P. R., Kleiner, A. C., Roberts, C., Ross, R. B., \& Smith, B. J. (1994). The fifth discipline fieldbook: Strategies and tools for building a learning organization. New York: Currency, Doubleday.

Sinkula, J. M., Baker, W. E., \& Noordewier, T. (1997). A framework for market-based organizational learning: Linking values, knowledge, and behavior. Journal of the Academy of Marketing Science, 25(4), 305-318. https://doi. org/10.1177/0092070397254003 
Sirén, C., Hakala, H., Wincent, J., \& Grichnik, D. (2017). Breaking the routines: Entrepreneurial orientation, strategic learning, firm size, and age. Long Range Planning, 50(2), 145-167. https://doi.org/10.1016/j.lrp.2016.09.005

Slater, S. F., \& Narver, J. C. (1995). Market orientation and the learning organization. Journal of Marketing, 59(3), 63-74. https://doi.org/10.2307/1252120

Sok, P., O'Cass, A., \& Sok, K. M. (2013). Achieving superior SME performance: Overarching role of marketing, innovation, and learning capabilities. Australasian Marketing Journal, 21, 161-167. https://doi.org/10.1016/j.ausmj.2013.04.001

Swee Lin Tan, C., Smyrnios, K. X., \& Xiong, L. (2014). What drives learning orientation in fast growth SMEs? International Journal of Entrepreneurial Behavior \& Research, 20(4), 324-350. https://doi.org/10.1108/ IJEBR-02-2013-0032

Tajeddini, K., Altinay, L., \& Ratten, V. (2017). Service innovativeness and the structuring of organizations: The moderating roles of learning orientation and inter-functional coordination. International Journal of Hospitality Management, 65, 100-114. https://doi.org/10.1016/j.ijhm.2017.06.010

Teece, D. J., Pisano, G., \& Shuen, A. (1997). Dynamic capabilities and strategic management. Strategic Management Journal, 18(7), 509-533. https://doi.org/10.1002/(sici)1097-0266(199708)18:7<509::aid-smj882>3.0.co;2-z

Torres, A. \& Jasso, J. (2009). Naturaleza y crecimiento de las empresas: la dinámica innovadora en las pymes de México. En Dutrénit, G. Sistemas regionales de innovación: un espacio para el desarrollo de las PYMES, 55-80. Uruguay: Textual.

Torres, A. (2006). Aprendizaje y construcción de capacidades tecnológicas. Journal of Technology Management \& Innovation, 1(5), 12-24.

Walter, A., Auer, M., \& Ritter, T. (2006). The impact of network capabilities and entrepreneurial orientation on university spin-off performance. Journal of Business Venturing, 21(4), 541-567. https://doi.org/10.1016/j. jbusvent.2005.02.005

Wang, E. T. G., \& Wei, H. (2005). The importance of market orientation, learning orientation, and quality orientation capabilities in TQM: An example from Taiwanese software industry. Total Quality Management \& Business Excellence, 16(10), 1161-1177. https://doi.org/10.1080/14783360500236270

Wang, C. L., \& Ahmed, P. K. (2007). Dynamic capabilities: A review and research agenda. International Journal of Management Reviews, 9(1), 31-51. https://doi.org/10.1111/j.1468-2370.2007.00201.x

Wang, Y. L. \& Ellinger, A. D. (2011) Organizational learning: Perception of external environment and innovation performance. International Journal of Manpower, 32(5/6), 512-536. https://doi.org/10.1108/01437721111158189 